\title{
VIOLENCIA EN INSTITUCIONES PENITENCIARIAS DEFINICIÓN, MEDICIÓN Y EXPLICACIÓN DEL FENÓMENO
}

\author{
Nicolás Trajtenberg y Olga Sánchez de Ribera
}

\section{Resumen}

La violencia en prisión es un problema fundamental para todos los sistemas penitenciarios del mundo. Compromete los derechos humanos, la seguridad $y$ la vida de los internos $y$ funcionarios, afecta la efectividad de los programas y puede incrementar la reincidencia y los costos sociales. Sin embargo, ha sido menos investigada que otras formas de violencia y la escasez de estudios es más grave en los contextos en los que más se necesitan. El objetivo de este artículo es presentar una sistematización de la literatura especializada sobre la violencia en prisiones para discutir sus principales problemas conceptuales y metodológicos. Se presentan los problemas de la definición de la violencia en el ámbito penitenciario; se examinan los principales desafíos enfrentados al intentar medir el fenómeno; se analizan las distintas explicaciones desarrolladas sobre el tema y se analizan modelos alternativos; y se cierra con un conjunto de conclusiones sobre los actuales desafíos para la investigación.

Palabras clave: violencia, prisión, medidas, definición, teorías.

\section{Abstract}

Prison violence. Definition, assessment and explanation

Prison violence is a fundamental problem for all prison systems in the world. It compromises the human rights, security, and integrity of the lives of the inmates and prison officers, affects the effectiveness of programs, recidivism and economic costs in society. Despite its relevance, prison violence has been little studied and the scarcity of studies becomes particularly serious in contexts where knowledge is more necessary. The objective of this study is to revise the specialized literature on violence in prisons to discuss its most relevant conceptual and methodological problems. Firstly, the violence definition problem in the penitentiary settings will be discussed. Secondly, the main challenges when attempting to measure the phenomenon will be examined. Thirdly, we will revise different theories and explanations of violence and alternative models. Finally, we make some conclusions regarding challenges for future research.

Keywords: violence, prison, assessment, theories.

\footnotetext{
Nicolás Trajtenberg: Profesor asociado de la Facultad de Ciencias Sociales de la Universidad de la República (Uruguay). Tiene una maestría en Criminología (Universidad de Oxford) y un doctorado en Criminología (Universidad de Cambridge). Integra el Núcleo de Análisis de la Criminalidad y la Violencia. ORCID iD: https://orcid.org/0000-0002-4451-3874

E-mail: nicolas.trajtenberg@cienciassociales.edu.uy

Olga Sánchez de Ribera: Investigadora postdoctoral en la Facultad de Psicología de la Universidad de la República (Uruguay). Tiene una maestría y un doctorado en Criminología por la Universidad de Cambridge.

ORCID iD: https://orcid.org/0000-0003-0344-3280

E-mail: olgasrdc@gmail.com
} 


\section{Introducción}

La violencia entre presos y entre presos y funcionarios es un problema fundamental para todos los sistemas penitenciarios del mundo. Este hecho no debería sorprender dado que las prisiones tienden a concentrar en un mismo espacio una cantidad considerable de individuos con potencial para la violencia (Schenk y Fremouw, 2012). Los datos muestran que la violencia forma parte de las instituciones penitenciarias y es sufrida no solo por internos (Ireland, 1999; Sorensen y Cunningham, 2010), sino también por el personal penitenciario (Sorensen, et al., 2011; Lahm, 2009). Sobre este problema es difícil tener estimaciones precisas y confiables, pero algunos estudios nos dan una aproximación de su magnitud.

Un informe sobre las condiciones de prisión europeas (Modvig, 2014) mostró que un $25 \%$ de los encarcelados sufre violencia física; casi un $5 \%$ sufre algún tipo de violencia sexual; casi un $2 \%$ reconoce haber sido violado; y la tasa de ataques físicos sufridos por los hombres en las prisiones es dieciocho veces mayor que la correspondiente a la población general (ver también Wolff, et al., 2008). Los informes sobre prisiones en Estados Unidos también señalan que la tasa de violencia y la probabilidad de sufrir victimizaciones violentas (salvo homicidio) son significativamente más altas en el ámbito penitenciario que en la población en general (Steiner y Cain, 2016).

En Latinoamérica, un estudio comparativo encontró que en Argentina un $21,2 \%$ de los presos afirma haber recibido golpes dentro de la cárcel y se observan cifras similares en Chile $(25,6 \%)$, algo más bajas en México $(16,5 \%)$ y Perú $(15,1 \%)$ y mucho más bajas en Brasil $(4,6 \%)$ y El Salvador (3,5\%). De los internos victimizados, en Chile el 66\% indica haber sido golpeado por el personal penitenciario. Una situación similar se encuentra en El Salvador (61,5\%) y Argentina (68,2\%), mientras que en México (63\%) y Perú $(47,7 \%)$ se responsabiliza sobre todo a otros internos (Sánchez y Piñol, 2015; ver también Bergman, 2015).

Si bien en Uruguay casi no existe investigación en la materia, hay un antecedente que analiza los datos del censo penitenciario de 2010 y señala que más del $25 \%$ de las personas encuestadas había sufrido malos tratos por parte del personal penitenciario, pero solo uno de cada cuatro realizaron denuncias (Juanche, 2012). Además, las cifras de homicidios son elocuentes: en 2017, la tasa de homicidios cada 100.000 habitantes en prisiones era de 
154, veinte veces mayor que la tasa nacional (8,1 cada 100.000) (Comisionado Parlamentario para el Sistema Penitenciario, 2017). ${ }^{1}$

En principio, dada la naturaleza de organizaciones tan especiales como las instituciones penitenciarias y, en este caso, las localizadas en la región latinoamericana, la violencia en prisiones, lejos de ser un hecho sorprendente, es una consecuencia natural. Hay una serie de características que permiten inferir que la violencia se maximiza en este tipo de escenarios: I) la concentración de una elevada proporción de individuos con antecedentes violentos; II) la infraestructura con carencias y con servicios y equipamiento de seguridad inadecuados o no adaptados; III) los recursos humanos inadecuados, con elevadas proporciones de funcionarios con escasa preparación, capacitación y motivación para realizar el trabajo y con débiles sistemas de supervisión y evaluación; y IV) los problemas de transparencia, visibilización y rendición de cuentas que impiden identificar las desviaciones e irregularidades que ocurren en el interior de los centros penitenciarios (Matthews, 2012; Gambetta, 2009).

La violencia en prisión implica un serio problema normativo para nuestras sociedades, tanto desde el punto de vista deontológico como desde la perspectiva consecuencialista. Por un lado, la violencia en prisión afecta los principios de justicia básicos en términos de derechos humanos claves. La dignidad, la seguridad y la integridad de la vida de personas que residen en las prisiones se ven seriamente amenazadas. Por otro lado, si nos centramos en las consecuencias o efectos, la violencia penitenciaria involucra una serie de importantes costos para la población penitenciaria, sus familias, la sociedad y el Estado. No solo implica múltiples costos directos de salud (enfermedades, discapacidad, salud mental), psicológicos y emocionales, sino también costos más intangibles, como la reducción de la moral y la motivación y el debilitamiento de la confianza y la legitimidad de la organización (Cooke, Wozniak y Johnstone, 2008; Gadon, Johnstone y Cooke, 2006; Steiner, Butler y Ellison, 2014). La violencia penitenciaria también puede afectar el desarrollo y la integridad de los programas de trabajo, educación y tratamiento (Auty, Cope y Liebling, 2017) e, incluso, algunos trabajos recientes muestran que el tipo de clima moral y social en la prisión está asociado con la reincidencia de los internos cuando son liberados (Auty y Liebling, 2019). En definitiva, las instituciones penitenciarias violentas son más complejas de gestionar y dirigir, y son mucho más costosas si se las compara con las instituciones seguras y con un ambiente de trabajo positivo (Modvig, 2014). Por otra parte, la existencia

1 Es importante ser cautos con estas comparaciones, ya que las tasas de homicidios en poblaciones de menos de 100.000 habitantes son particularmente sensibles a pequeños cambios. No obstante, una mirada de más largo plazo (ver el trabajo de Vigna y Sosa [2019] en este número) confirma esta marcada desproporción entre las tasas de homicidio para la población general y para la población penitenciaria. 
de altos niveles de violencia interpersonal en el interior de las prisiones aumenta la probabilidad de que los internos reincidan al salir en libertad (Mooney y Daffern, 2015; Trulson, Delisi y Marquart, 2011).

Paradójicamente, pese a que los sistemas penitenciarios en estado más crítico y con mayores niveles de violencia se encuentran en los países en desarrollo (Jackson, Heard y Fair, 2017), la investigación en esta temática está concentrada en su mayoría en Norteamérica y el Reino Unido (ArbachLucioni, Martínez-García y Andrés-Pueyo, 2012; Sanhueza, Smith y Valenzuela, 2015). Sabemos menos sobre la violencia penitenciaria que ocurre en lugares donde este conocimiento es más crucial y relevante para la toma de decisiones de política pública. Existe un importante déficit de investigación cross-cultural (transcultural) de violencia penitenciaria en sociedades en desarrollo. Un caso paradigmático es América Latina, donde se combinan tres condiciones desafortunadas: I) prisiones con serios problemas de infraestructura, hacinamiento, personal y violencia carcelaria (De León Villalba, 2018; Jackson, Heard y Fair, 2017); II) gobiernos, políticas y sistemas penales que favorecen el aumento de la población privada de libertad con mayor tiempo de reclusión, lo que aumenta la presión en dichas instituciones con el consiguiente aumento de la violencia en ellas (Dammert y Zúñiga, 2008); III) sistemas de información débiles, poco fiables e incompletos; y IV) la escasa y débil investigación existente basada en estudios de caso o estudios comparativos descriptivos, salvo alguna excepción puntual (por ejemplo, para el caso chileno, Sanhueza, Smith y Valenzuela).

\section{La definición del fenómeno}

Pese a la relevancia de la violencia en la prisión, su medición constituye un serio problema. Es un fenómeno multidimensional sobre cuya definición existe mucha controversia, así como sobre qué variantes y componentes debería incluir (Sorensen y Cunningham, 2007; Wolff, Shi y Bachman, 2008). En realidad, el concepto de violencia en prisión presenta los mismos problemas conceptuales que el concepto general de violencia, para el que también existen múltiples definiciones y desacuerdos entre expertos y organismos internacionales.

La definición de la Organización Mundial de la Salud establece que es "el uso intencional de la fuerza o el poder físico, de hecho o como amenaza, contra uno mismo, otra persona o un grupo o comunidad, que cause o tenga muchas probabilidades de causar lesiones, muerte, daños psicológicos, trastornos del desarrollo o privaciones" (Organización Mundial de la Salud, 2002). A la hora de clasificar los tipos de violencia hay dos criterios claves manejados por los organismos internacionales y los expertos (Imbush, Misse y Carri, 2011; Organización Mundial de la Salud, 2002; Oficina de Naciones Unidas contra la Droga y el Delito, 2012). El primer criterio hace referencia 
al objeto de la violencia, es decir, a la víctima. Con base en este criterio, se distinguen tres tipos de violencia: a) autoinfligida, cuando el perpetrador es la víctima, ya sean comportamientos suicidas o autolesiones; b) interpersonal, es la sufrida a manos de una o pocas personas; ${ }^{2}$ y c) colectiva, que es la infligida por grandes grupos o instituciones (por ejemplo, el Estado, grupos políticos, grupos paraestatales, organizaciones terroristas, etcétera). ${ }^{3} \mathrm{El}$ segundo criterio hace referencia a la naturaleza del comportamiento, es decir, a de qué manera la víctima se ve afectada por el comportamiento violento, lo que puede incluir aspectos físicos, sexuales, psíquicos e inclusive asociados a la negligencia o el descuido.

Si bien esta definición, con estos dos criterios de base, es un punto de arranque muy importante para la conceptualización de la violencia en general y en particular para el ámbito penitenciario, presenta los siguientes problemas.

En primer lugar, es un concepto que, si bien apela a actos intencionales o deliberados, genera de todos modos desafíos importantes a la hora de establecer en qué condiciones es adecuado atribuirla. En otras palabras, requiere un juicio sobre los procesos motivacionales que llevan a generar las acciones, algo que suele ser complicado de lograr porque las diferencias entre acciones deliberadamente violentas y acciones que generan daño por negligencia son graduales y no categóricas (Eisner, 2009; ver también Sheeran, 2012; Rosenberg, 2012). Además, como señalan Krug y colegas (2003), aun si la violencia exigiese intencionalidad, el hecho de que exista intención de usar la fuerza no necesariamente implica que haya intención de generar daño. Una persona puede cometer un acto que es considerado peligroso y que posiblemente genere efectos negativos sobre la salud de una potencial víctima, pero sin tener ella misma esa percepción.

En segundo lugar, la inclusión del término poder hace más extensiva la definición de violencia, al permitir integrar actos más difusos que pueden ser el resultado de asimetrías y relaciones de poder que involucran desde las amenazas y la intimidación hasta aquellos menos claros como el descuido o los actos por omisión (Krug, et al., 2003; Organización Mundial de la Salud, 2002). Esta definición más extensiva complejiza la identificación y la medición de la intencionalidad, sobre todo cuando los hechos tienen

2 A su vez, esta categoría se divide en dos subcategorías: a) la violencia familiar o de pareja, que se sufre por lo general en el hogar y ocurre entre miembros de familia, y no tiene relevancia para el contexto penitenciario; y b) la violencia comunitaria, que ocurre entre personas que no tienen vínculos de parentesco y que pueden tener algún grado de conocimiento o no, y es particularmente relevante para la violencia penitenciaria, ya que puede tener lugar en establecimientos como el trabajo, la escuela o las prisiones (Organización Mundial de la Salud, 2002).

3 En nuestro caso, se define cuando existe un ejercicio sistemático de la violencia que tiene origen en las autoridades y es obedecido por los diferentes tipos de funcionarios de la institución penitenciaria. 
lugar en organizaciones complejas como las instituciones penitenciarias donde, en muchos casos, los individuos se comportan de manera violenta siguiendo órdenes o amenazas de otros y donde, por ende, puede haber una gran distancia entre el autor intelectual y el autor material que genera el daño (Eisner, 2009).

En tercer lugar, la definición incorpora dos aspectos de la violencia muy complejos de identificar y medir: a) los tipos de actos o comportamientos a ser considerados violentos, incluyendo actos públicos o privados, activos o reactivos, delictivos o no delictivos; y b) las consecuencias o efectos de la violencia, con el fin de incluir no solo los actos de violencia que generan daños físicos y visibles, sino también aquellos que por vías más indirectas generan daños emocionales, psíquicos, y sociales, tanto inmediatos como latentes, que pueden manifestarse mucho tiempo después de que tuvo lugar el maltrato (Krug, et al., 2003; Organización de Naciones Unidas contra la Droga y el Delito, 2014).

Estas dificultades conceptuales y metodológicas complejizan la posibilidad de llegar a un acuerdo entre los expertos en torno a qué es exactamente la violencia penitenciaria, lo que conlleva a que en ocasiones se use el mismo término para referir a comportamientos muy heterogéneos.

Como señala Modvig (2014), en las instituciones penitenciarias las autoridades tienen la obligación de proteger a las personas privadas de libertad de cualquier tipo de violencia, excepto el uso proporcionado y necesario de la fuerza para procedimientos de seguridad, y se entiende por violencia comportamientos tan variados como: ataques físicos y lesiones, homicidios, abusos sexuales y violaciones, distintos tipos de agresiones psicológicas, amenazas, humillaciones, bullying, conductas indebidas, suicidios o autolesiones (Ireland, 2002). Si bien una parte importante de la violencia ocurre entre personas privadas de libertad (y, de hecho, es en lo que se concentra casi toda la investigación existente), los roles de víctima y victimario pueden variar y la violencia puede ser ejercida no solo entre internos, sino también: de funcionarios a internos y de internos a funcionarios. Por ello también es importante incluir tanto las formas colectivas de violencia ejercidas para enfrentar a las autoridades, como motines, toma de rehenes o fugas, como las formas de violencia institucional más invisibles y veladas, que incluyen el uso excesivo e inadecuado de fuerza, la tortura, los malos tratos, los abusos sexuales y psicológicos, entre otras (Matthews, 2012; Modvig, 2014).

\section{Medición del fenómeno}

Medir todas estas formas de violencia penitenciaria con validez y confiabilidad no es tarea fácil y las estimaciones existentes en general subestiman el fenóme- 
no (Wolff, et al., 2007). ${ }^{4}$ Como en otras formas de violencia, existe una cifra negra de la violencia penitenciaria que no se refleja en las estadísticas y queda oculta, sobre todo por su carácter ilegal, es decir, porque quien la comete no quiere que se conozca por su carácter ilegítimo, que puede llevar a sanciones (Aebi, 2008; Maguire, 2012). Cuando la violencia es perpetrada por funcionarios o por presos asociados a funcionarios o autoridades, hay menos probabilidades de que el evento violento sea conocido y reportado. En el contexto penitenciario las motivaciones de las víctimas para denunciar son particularmente débiles, ya que existen fuertes códigos culturales respecto a los "soplones" y miedo a las represalias. Esto tiene un efecto disuasor relevante, máxime teniendo en cuenta que, como está restringida la libertad de circulación, hay mayor certeza de sufrir las represalias (Byrne y Hummer, 2007; Wolff, et al., 2007).

Las víctimas de violencia penitenciaria pueden no reportar la violencia sufrida por varias razones, algunas de las cuales han sido señaladas por los estudios sobre victimización: muchas veces los episodios de violencia (y más los de menos gravedad) no son denunciados porque la víctima ni siquiera percibe que hubo violencia, dado que la ha aceptado como parte normal y natural de la vida cotidiana del encierro; en otros casos, aun siendo consciente de la violencia sufrida, no tiene confianza en el sistema penal, en las autoridades o en los funcionarios y considera que la denuncia no tendrá ningún efecto positivo (Aebi, 2008; Kidd y Chayet, 2010; para el caso uruguayo, ver Juanche, 2012).

En la literatura se mencionan varias fuentes de información sobre el fenómeno. La primera son las estadísticas oficiales o datos secundarios provenientes de las instituciones penitenciarias. Estas estadísticas tienen una serie de problemas de fiabilidad similares a los que ocurren con las estadísticas oficiales de violencia fuera de prisión. Por un lado, dependen del reporte de la víctima, que está sesgado por su interpretación, por el miedo a represalias y por la desconfianza en las autoridades (Schenk y Fremouw, 2012; Wolff, et $a l ., 2007)$. Por otro lado, la detección, el registro y la interpretación realizados por los funcionarios penitenciarios están fuertemente determinados por la escasa uniformidad y estandarización de los procesos y por factores subjetivos como la motivación y la voluntad del personal (Aebi, 2008; Ireland, 2002).

En términos de validez, las estadísticas oficiales muestran un panorama incompleto y muy sesgado del problema, ya que en general se centra en las

4 La medición del fenómeno busca evaluar qué proporción de individuos dentro del establecimiento penitenciario sufre o perpetra los distintos tipos de violencia. Al igual que con las medidas de violencia o victimización generales (Aebi, 2008), la clave es el rango temporal de interés: en algunos casos, interesa saber si alguna vez en su vida el individuo participó en algún tipo de incidente (prevalencia de vida); en otros casos, se busca estimar la participación en tiempos más recientes: prevalencia de los últimos doce o seis meses o, inclusive, prevalencia del último mes. Para un conocimiento más detallado de frecuencia o intensidad del fenómeno, se usan las medidas de incidencia en los últimos doce meses o seis meses o en el último mes. 
variantes más graves de violencia, como el homicidio, las violaciones y los ataques físicos, dejando de lado otros tipos de violencia, como el bullying o formas más simbólicas de agresión (Bottoms, 1999; Ireland e Ireland, 2000). La consecuencia natural de estos sesgos es que la comparabilidad del fenómeno entre distintas instituciones penitenciarias se vuelve muy compleja debido al carácter idiosincrático que poseen los procedimientos y registros de las distintas instituciones (Ireland, 2002). Además, las fuentes oficiales tienden a recoger escasa o incompleta información sobre múltiples aspectos de la persona (psicológicos, morales, de crianza, familiares, educativos, laborales, sobre grupos de pares, barriales, etcétera) que son relevantes para explicar el fenómeno.

Una alternativa a los datos oficiales es el uso de datos primarios, entre los cuales se destacan los autorreportes de personas privadas de libertad. Sin embargo, hay que tener en cuenta dos aspectos: por un lado, la forma en la que se aplica el cuestionario, que puede ser por entrevista o autoadministración, $\mathrm{y}$, por otro, el carácter de las escalas aplicadas, es decir, si es un formulario centrado exclusivamente en medir violencia o si la violencia es medida en el marco de una escala más general. Respecto a la forma, el uso de entrevistas estructuradas o semiestructuradas permite generar información detallada de los eventos o episodios violentos en prisión, pero presenta los siguientes inconvenientes: las entrevistas son muy costosas en términos de tiempo, por lo que suelen aplicarse a un número reducido de internos; se ven afectadas por los sesgos de memoria del entrevistado; y no aseguran el anonimato, lo que tiene consecuencias en la validez de la información obtenida (Connell y Farrington, 1996; Ireland, 2002; Rocheleau, 2013).

El método de cuestionario autoadministrado mediante un listado de ítems (checklist) de comportamientos agresivos específicos ${ }^{5}$ es de los formatos más empleados en la literatura porque permite superar alguno de los inconvenientes antes mencionados: disminuye la variabilidad de interpretación del encuestado, permite optimizar los tiempos, permite la aplicación a muestras relativamente extensas, permite mantener el anonimato y permite lograr niveles de validez y confiabilidad aceptables (Ireland y Archer, 1996; Ireland, 2002). Además de estos, en criminología se utilizan instrumentos más específicos que apuntan a medir el clima institucional penitenciario e incluyen ítems relacionados con la percepción de inseguridad, el miedo o las victimizaciones sufridas por los internos. Ejemplos de ellos son la Correctional Institutions Enviroment Scale, de Moos (1975), o la

5 Una alternativa metodológica que ha resultado ser menos adecuada metodológicamente, pero que también ha sido empleada en algunas investigaciones es usar cuestionarios autorreportados preguntándoles a los internos si han sufrido o si han perpetrado comportamientos de bullying. Un problema serio de este tipo de planteos es que la connotación negativa del término favorece el subreporte y existe mucha heterogeneidad en la interpretación que los encuestados hacen de él (Connell y Farrington, 1996). 
más reciente Measuring the Quality of Prison Life, de Liebling, Hulley y Crewe (2011). No obstante, si se quiere tener una estimación más completa del fenómeno, los instrumentos más apropiados son las escalas centradas en una amplia serie de malas conductas, conductas violentas, bullying y abusos por terceras partes. Ejemplos de estos instrumentos son el cuestionario autoinformado de Connell y Farrington (1996), la escala de victimización física de Wolff, et al. (2007) y la Direct and Indirect Prisoner Behaviour Checklist, de Ireland (1999).

Aunque este conjunto de instrumentos permite mejorar las estimaciones de violencia, no está exento de limitaciones. Primero, existen problemas de deseabilidad social y tendencia a no reportar algunos comportamientos particularmente graves o vergonzosos. Dados los fuertes códigos y subculturas carcelarias, este sesgo puede influir de manera decisiva a la hora de no reportar la victimización más que la perpetración (Connell y Farrington, 1996). Segundo, los múltiples problemas de sesgos de memoria afectan la validez de la información recogida, sobre todo cuando se refiere a eventos más distantes en el tiempo y menos graves (Junger-Tas y Haen Marshall, 1999; Schenk y Fremouw, 2012). Tercero, pese al mayor anonimato respecto a otros métodos, igual se observan niveles elevados de rechazo a responder y cierta negación o rechazo en relación con reconocer haber cometido actos violentos (Dyson, Power y Wozniak, 1997). Cuarto, estas escalas no permiten obtener información relevante referida tanto a la identificación de la naturaleza del perpetrador como a la intensidad o severidad de la violencia ejercida ni de sus consecuencias (Ireland, 2002). Por último, estos instrumentos tienden a centrarse en factores individuales y dejan de lado factores claves y difíciles de medir relacionados con el funcionamiento y la gestión institucional, que son decisivos para elaborar programas efectivos de prevención de la violencia carcelaria (Cooke, Wozniak y Johnstone, 2008). ${ }^{6}$

Como respuesta a varias de estas limitaciones, se han desarrollado herramientas mixtas que permitan evaluar de forma más sistemática los factores de riesgo, tanto individuales como estructurales y de gestión institucional, mediante la triangulación de datos oficiales de internos y profesionales, entrevistas en profundidad y observación no participante. Un ejemplo paradigmático es Promoting Risk Intervention by Situational Management (PRISM), desarrollada por Johnstone y Cooke (2008) que explora las interacciones entre cinco áreas principales: 1) la historia de violencia institucional del centro penitenciario: niveles previos de violencia, diversidad de violencia, escaladas, etcétera; 2) la gestión individual: evaluaciones de violencia individual, intervenciones para reducir la

6 Algunas alternativas poco empleadas son los reportes de pares (en algunos casos, de otros internos y, en otros, de funcionarios) o los grupos motivacionales o foco (Connell y Farrington, 1996; Ireland, 2002). 
violencia, cómo se mezclan las distintas poblaciones; 3 ) aspectos físicos y de seguridad: medidas de seguridad, entorno físico y recursos disponibles; 4) características del personal: tipo de reclutamiento, formación y competencias, experiencias, estilo y enfoque, moral, etcétera; 5) aspectos más institucionales u organizacionales: ethos y prioridades, estructura organizacional, liderazgo y gestión en materia de violencia, políticas y programas empleados para reducir la violencia y responsividad y flexibilidad para adaptarse al conflicto y al cambio (Cooke y Wozniak, 2010).

En definitiva, la experiencia de las últimas décadas indica que el fenómeno de la violencia penitenciaria es un desafío metodológico formidable. Las diferentes formas de medir la violencia penitenciaria tienen múltiples ventajas y desventajas en términos de validez y confiabilidad. Parece claro que obtener una medición mínimamente fidedigna y no sesgada solo es posible si combinamos y triangulamos varias de estas medidas.

\section{Explicación del fenómeno}

A pesar de la relevancia de este fenómeno y la necesidad de estudiar su naturaleza y causas para poder intervenir de manera eficaz, la violencia en prisión ha sido mucho menos estudiada y explicada que otras formas de violencia en la sociedad. No obstante, en las últimas décadas se han desarrollado algunas teorías que ayudan a entenderla. En un sentido amplio, la literatura se divide en dos grandes teorías: de importación y de deprivación. Ambas se describen a continuación.

La teoría de la importación o continuidad establece que son las condiciones y características que el interno trae consigo desde antes de ser privado de la libertad las que tienen influencia en sus comportamientos violentos en prisión (Irvwin y Cressey, 1962). Determinadas características hacen que ciertos individuos tengan más dificultades para adaptarse y comportarse en el entorno penitenciario. Entre estas destacan: factores individuales (por ejemplo, trastornos mentales y de personalidad, rasgos psicopáticos o antisociales, baja autoestima, alta impulsividad, antecedentes de abuso de drogas, antecedentes delictivos y penitenciarios, tener valores y pensamientos delictivos o desviados); factores socioeconómicos $y$ demográficos (ser varón y joven, pertenecer a una minoría racial, estatus de marginalidad y vulnerabilidad social, bajo nivel educativo, ausencia de trabajo); y factores sociocomunitarios (no estar en pareja, redes familiares, sociales y comunitarias débiles, pertenecer a pandillas [gangs] y redes o subcultura delictivas) (Arbach-Lucioni, Martínez-García y Andrés-Pueyo, 2012; Bottoms, 1999; Cunningham, et al., 2010; Gendreau, Goggin y Law, 1997; Mears, et al., 2013; Walters, 2003; Walters, 2011). Por tanto, esta teoría asume una continuidad en el sentido de que el individuo que es violento fuera de la prisión lo es también dentro de ella. 
La teoría de la privación o institucional (o de la deprivación), de Sykes (1958), plantea que las causas de la violencia se localizan en la propia privación de libertad y en la experiencia de sufrimiento que implican la falta de libertad, autonomía e independencia y el limitado acceso a bienes y servicios. En este entorno brutal y de carencias, los individuos privados de libertad buscan medios ilegítimos alternativos, y muchas veces violentos, para satisfacer o compensar la privación de estas necesidades. Sykes asume cierta discontinuidad, ya que muchos individuos que no utilizaban la violencia en la comunidad, en la cárcel se vuelven violentos como una forma de adaptación y supervivencia a la cultura carcelaria. En esta teoría se distinguen dos mecanismos individuales de adaptación: el primero, más irracional, tiene que ver con que el estrés y la frustración generados por la privación provocan reacciones violentas frente a otros presos y funcionarios penitenciarios; el segundo, más racional, se vincula con que los individuos se enfrentan a un entorno muy violento, competitivo y con escasez de recursos, donde saben que tienen que emplear vías ilegitimas y violentas para lograr sus objetivos y satisfacer sus necesidades.

Además, en esta teoría se distinguen dos aspectos importantes: los estructurales de la prisión y aquellos más asociados la gestión de los sistemas penitenciarios. Entre los factores de orden más estructural, la evidencia empírica indica que la mala conducta y la violencia carcelaria están asociadas con los niveles de seguridad de los centros (más concretamente, con las cárceles de máxima seguridad), su tamaño, su diseño arquitectónico, los niveles de hacinamiento, las agendas de visitas, las experiencias de victimización, el estrés y las tensiones experimentados y la percepción de la legitimidad de los funcionarios y autoridades (Blevins, et al., 2010; DeLisi, Berg y Hochstetler, 2004; McCorkle, Miethe y Drass, 1995; Pierce, et al., 2018; Porporino, Doherty y Sawatsky, 1987). La evidencia indica que los actos violentos no se distribuyen de modo homogéneo en el espacio y el tiempo penitenciario. No solo existen «puntos calientes» (hot spots) de la violencia penitenciaria en determinadas áreas o módulos, sino que también se concentran en determinados días (viernes y fines de semana) y en ciertos horarios (vespertinos y nocturnos) (Porporino, Doherty y Sawatsky, 1987). También se ha observado que los individuos que cumplen condenas largas o sin acceso a salidas tienden a cometer menos violencia (Cunningham, Reidy y Sorensen, 2005) y que la violencia tiende a aparecer más en el inicio de la estadía en prisión (Bottoms, 1999; Cunningham y Sorensen, 2007; Rocheleau, 2013). Entre los componentes de gestión asociados a la violencia, los estudios refieren a estilos de gestión abusivos y basados en el control y sanciones punitivas; los niveles de supervisión, la cantidad y la calidad del personal; la satisfacción y el estrés laboral del personal; el nivel de conocimiento y habilidades de los directores de prisión; la existencia de programas de tratamiento; la mezcla de internos con diferentes niveles de riesgo; la relación entre los internos y el personal (Cooke, Wozniak 
y Johnstone, 2008; Cochran, 2012; Dilulio, 1987; Gadon, Johnstone y Cooke, 2006; Homel y Thomson, 2005).

La investigación reciente muestra que la explicación de la violencia debe ser integral y hacer interactuar, simultáneamente, tanto las características previas al ingreso a prisión como las características estructurales y de gestión, que determinan cómo es la experiencia de privación de libertad (Cooke y Wozniak, 2010; Ireland, 2012; McGuire, 2018; Steiner, Butler y Ellison, 2014).

Una forma de visualizar esta integración es aplicar el esquema que utiliza Redondo (2008) y su teoría del triple riesgo delictivo, que realiza una síntesis parsimoniosa de tres categorías de factores de riesgo (personales, sociales y oportunidades para desviarse) y predice que los individuos con mayor riesgo de conducta antisocial son los que presentan combinaciones más negativas de las tres categorías de riesgo (Redondo, 2008). De manera análoga, el riesgo de violencia intracarcelaria podría sintetizarse y predecirse a partir de tres conjuntos de factores de riesgo asociados a: a) aspectos individuales propios de la teoría de la continuidad, b) aspectos estructurales y c) aspectos de gestión, los dos últimos asociados a la teoría de la discontinuidad.

\section{Figura 1. Factores explicativos de la violencia intracarcelaria}

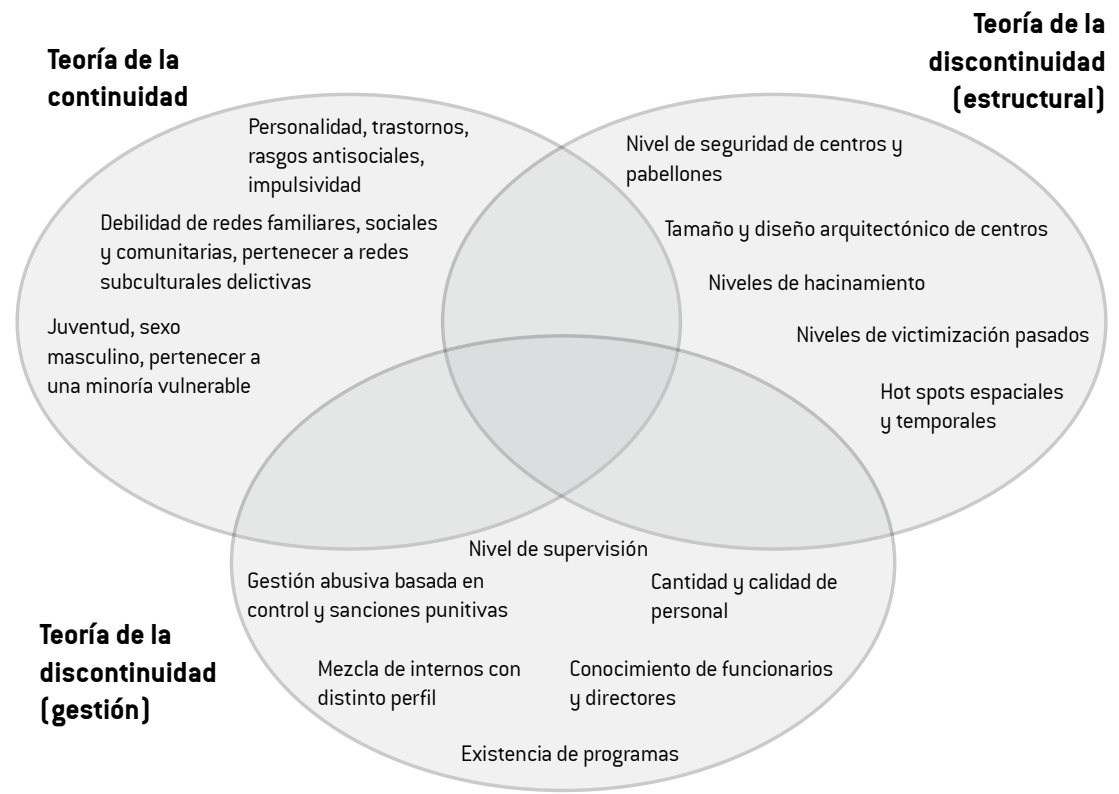

Fuente: Elaboración propia, adaptado de Redondo (2008).

De manera reciente, se ha buscado enriquecer las explicaciones de los comportamientos agresivos en prisión con teorías criminológicas de la 
violencia. Por ejemplo, Blevins y colegas (2010) argumentan que se puede utilizar la teoría de la tensión, de Agnew (2012), para reconceptualizar e integrar las teorías de la deprivación y la importación antes señaladas. La teoría de la tensión de Agnew establece que la experiencia de sufrir muchas y muy intensas tensiones provoca emociones negativas, lo que, a su vez, genera una presión correctiva que puede desembocar en comportamientos agresivos. Según Blevins y colegas (2010), la teoría de la tensión y la teoría de la deprivación identifican que los individuos privados de libertad deben adaptarse a un entorno que elimina los estímulos positivos y los enfrenta a estímulos negativos, haciendo muy improbable que puedan lograr objetivos valorados de manera positiva. Sin embargo, tomando la teoría de la importación, la reacción y la adaptación a las tensiones en prisión pueden no ser homogéneas y dependen, en cambio, de muchas características individuales y sociales de los individuos (personalidad, pares, relaciones de apoyo), así como de las estrategias disponibles para afrontar las tensiones.

Otra teoría general de la violencia que ha sido aplicada al contexto penitenciario es la teoría del control social (Hirschi, 1969; Sampson y Laub, 1993). Según los supuestos básicos de esta teoría, los individuos están naturalmente motivados a desviarse de las normas y, por lo tanto, la pregunta central es qué hace que algunos individuos no se desvíen. Y la clave, según estos autores, está en que los individuos que no se desvían presentan un vínculo y un control social informal más intenso con base en una serie de castigos y recompensas. Algunos estudios han mostrado que variables como la edad, la raza, el vínculo marital, tener hijos, la duración de la sentencia, el régimen penitenciario, la participación en programas de tratamiento y programas laborales pueden ser utilizadas como indicadores de vínculo con la sociedad convencional y con controles informales que revelan tener capacidad explicativa en la comisión o no de infracciones violentas y no violentas en el ámbito penitenciario, tanto para muestras de hombres (Wooldredge, Griffin y Pratt, 2001) como para mujeres (Steiner y Wooldredge, 2009). ${ }^{7}$

La teoría de la legitimidad, de Tyler (1990), que se enmarca dentro de las teorías del control social, también ha sido utilizada para explicar la violencia carcelaria. El propio Tyler y otros coautores han argumentado que el orden y la estabilidad en prisión, y por ende los menores niveles de violencia, dependen no tanto de la coerción y capacidad de control como de la percepción de legitimidad que los internos tienen de las autoridades

7 Algunos autores, como Steiner, Butler y Ellison (2014), ven un vínculo importante entre las teorías de gestión penitenciaria antes referidas y las teorías del control, en particular las del control administrativo, mediante las que se busca demostrar cómo algunas condiciones ambientales asociadas al debilitamiento del control administrativo y la desorganización institucional pueden generar conductas violentas en las prisiones (Steiner, 2009) o inclusive formas colectivas de violencia como motines (Useem y Reisig, 1999). 
y funcionarios del sistema penitenciario. Es decir, el orden y la ausencia de violencia en prisión se basan en que las personas privadas de libertad acepten la autoridad penitenciaria y se sientan obligadas a seguir sus normas y órdenes. Esta percepción de legitimidad depende, a su vez, de la percepción que tienen los presos del respeto y la equidad con los que son tratados por las autoridades y funcionarios (Jackson, et al., 2010).

Otra teoría criminológica general que ha sido aplicada con relativo éxito a la explicación de la violencia carcelaria es la teoría del autocontrol, de Gottfredson y Hirschi (1990). Según esta teoría, la capacidad de autocontrol de cada individuo es lo que determina que las personas puedan (o no) involucrarse en actividades desviadas y violentas. La falta de autocontrol se expresa principalmente en una mayor impulsividad, un temperamento más volátil, mayor egocentrismo y menor aversión al riesgo. Varios estudios han mostrado cómo algunos rasgos, en particular la aversión al riesgo y el temperamento volátil, permiten predecir la perpetración de infracciones y la victimización en prisión (Kerley, Hochstetler y Copes, 2009; Kerley, et al., 2011).

Por su parte, otros estudios han intentado aplicar las teorías de la victimización delictiva al contexto penitenciario. En particular, la teoría de actividades rutinarias (Cohen y Felson, 1979; Felson y Boba, 2010) y la teoría de los estilos de vida (Hindelang, Gottfredson y Garofalo, 1978). El argumento central es que los riesgos de victimización de las personas están asociados a las rutinas de circulación vinculadas a sus estilos de vida. En particular, ciertas actividades rutinarias determinan que aumente la victimización en ciertas áreas y ciertos momentos porque favorecen la confluencia de tres condiciones: objetivos adecuados (las víctimas), delincuentes motivados y ausencia de guardianes formales o informales. En el contexto penitenciario se ha observado cómo las actividades diarias de los internos determinan el nivel de presencia de guardianes (no solo de funcionarios o psicólogos, sino también de otros internos) y aumentan (o no) su exposición a situaciones de riesgo y a sufrir agresiones (Wooldredge y Steiner, 2013; 2014; Steiner, et al., 2017). Algunos autores han mostrado también que los niveles de victimización penitenciaria asociados al tipo de vigilancia varían entre cárceles, según el tipo de diseño arquitectónico, la tasa de funcionarios por presos, la presencia de tecnología de vigilancia (por ejemplo, cámaras) y las medidas de identificación y seguridad (Useem y Piehl, 2006).

Gambetta (2009) plantea una explicación de la violencia penitenciaria anclada en una teoría de la comunicación y del intercambio de señales. Según el sociólogo italiano, el vínculo entre violencia y comunicación es doble. Por un lado, hay un vínculo inverso, es decir, cuanto mayor es la reputación de un preso de ser violento, menor necesidad tiene de emplear la violencia. Por otro lado, hay un vínculo directo, es decir, en muchos casos la violencia se usa para comunicar la capacidad de ser violento y crearse así una reputación 
útil que permita prevenir ser victimizado en el futuro. Gambetta busca entender cómo se produce este doble vínculo entre violencia y comunicación en el contexto carcelario, donde los individuos deben comunicarse con restricciones y condiciones muy particulares: están obligados a convivir en espacios limitados con individuos sobre cuyas características y formas de proceder poseen muy poca información y el uso de la violencia es una ventaja competitiva para alcanzar bienes y servicios de difícil acceso, por mencionar algunas.

Inspirándose en la teoría del comportamiento agresivo de animales, de Krebs y Davies (1993), Gambetta plantea un modelo con cuatro hipótesis explicativas de la violencia penitenciaria asociadas a procesos comunicativos. La primera hipótesis es que las personas privadas de libertad buscan establecer sus posiciones en la jerarquía de violencia en prisión por medios comunicativos con el objetivo de generar (y observar en otros) señales creíbles que demuestren habilidad y disposición para ser violentos (capital violento). La segunda hipótesis es que cuando estas señales para conocer a los demás no sean accesibles o creíbles, hay más desafíos y violencia para establecer posiciones. Eso se traduce en dos corolarios más específicos. Por un lado, cuanto mayor capital violento posee un delincuente cuando llega a prisión, menos necesidad tiene de ejercer efectivamente la violencia (es decir, son los que tienen menos experiencia y menos habilidad en el uso de la violencia los que tienen que demostrarla por la vía de pelearse). Por otro lado, mucha violencia puede ser producto no de la falta de capital violento de los presos, sino de la ausencia de medios para poder señalizarlo con efectividad. Es decir, cuanto mejor es la información que circula sobre el capital violento de los presos, menos violencia interpersonal tiene lugar. La tercera hipótesis señala que los presos tienen una mayor probabilidad de involucrarse en comportamientos violentos cuando deben interactuar con otros presos sobre los que poseen poca o nula información. Por último, la cuarta hipótesis establece que, dado que hay una fuerte motivación de todas las personas privadas de libertad por conocer el capital violento de los otros presos y dado que pelear genera información útil y creíble sobre este capital (tanto para los contendientes como para otros internos), hay incentivos para desear que los otros se peleen entre sí, para recompensar a los que quieren pelear y castigar a los que lo evitan.

Más recientemente, algunos autores han planteado que se debería aplicar el paradigma del desistimiento del delito en el contexto penitenciario, según el cual explicar la reincidencia y el abandono del delito no son dos caras de una misma moneda (Sampson y Laub, 1993). Existen dos grandes paradigmas del desistimiento. Por un lado, la teoría de cambios objetivos, basados en el control social, según la cual el abandono del delito está asociado a cambios vitales relevantes o puntos de inflexión exógenos mediante los que se refuerza el vínculo con la sociedad, como obtener un 
trabajo, tener una pareja convencional o alistarse en el ejército (Sampson y Laub, 1993; Laub, Nagin y Sampson, 1998; Laub y Sampson, 2001). Por otro, la teoría de los cambios subjetivos plantea que la diferencia entre los que persisten en el mundo del delito y los que lo abandonan no está en las condiciones objetivas, sino en cambios internos, subjetivos, vinculados a la interpretación y la obtención de sentido del mundo que pueden llevar a los actores a buscar relaciones prosociales y oportunidades convencionales (Giordano, Cernkovich y Rudolph, 2002; Maruna, 2001). Uno de los pocos estudios recientes muestra que existe algo de respaldo empírico para la teoría de los cambios subjetivos, señalando un mayor abandono de la violencia penitenciaria en individuos privados de libertad que poseen más niveles de agencia, actitudes prosociales y resiliencia, en particular en los jóvenes. Estos cambios internos se ven acentuados cuando hay una atmósfera de trabajo positiva. Sorprendentemente, las mejoras en los vínculos comunitarios y los lazos sociales no son relevantes para entender el abandono de la violencia penitenciaria (Ellis y Bowen, 2017). ${ }^{8}$

\section{Conclusiones}

Pese a la relevancia y la gravedad de la violencia penitenciaria, este fenómeno ha sido muy desatendido por la investigación académica en comparación con otras formas de violencia. No obstante, en las últimas décadas se observa un interés creciente por él y por mejorar las formas que tenemos de definirlo, medirlo y explicarlo. La incorporación de datos primarios como complemento de los datos secundarios producidos por las instituciones penitenciarias es un punto de inflexión a la hora de generar estimaciones más confiables y válidas del fenómeno.

Un desafío metodológico clave es cómo continuar mejorando la combinación y comparación de las fuentes, en particular combinando no solo datos primarios y secundarios cuantitativos, sino también incorporando la triangulación con técnicas cualitativas, como han sugerido principalmente

8 Si bien el problema de la violencia penitenciaria es clave por las razones antes mencionadas (ver introducción), en ocasiones la disminución de la violencia podría ser una señal preocupante de un problema más invisible y velado asociado a un debilitamiento del Estado. Algunos estudios recientes sobre el caso brasileño son sintomáticos al respecto. Autores como Willis (2014) o Lessing (2017) han mostrado cómo en algunas ciudades brasileñas las políticas de encarcelamiento masivas y la segregación por afiliación a gangs, si bien permiten disminuir la violencia penitenciaria en el corto plazo, terminan generando el efecto perverso de favorecer el desarrollo y la expansión de esas gangs por dos vías: la consolidación interna por la vía del reclutamiento de adeptos en el interior de las prisiones y la extensión de su capacidad para explotar y corromper a funcionarios penitenciarios; la consolidación externa por la vía de extender su poder en las calles a través del control de los mercados ilícitos. En definitiva, la violencia se reduce en la prisión (y también en las calles) producto de acuerdos invisibles donde el poder estatal está en disputa y se ve obligado a ceder ante mafias y grupos de delito organizado (Willis, 2014; Lessing, 2017). 
Johnstone y Cooke (2008). Este es un desafío más general en la criminología, donde desde hace ya tiempo diversos autores evidencian la necesidad de triangular e integrar métodos cuantitativos y cualitativos (Bachman y Brent, 2014; Maruna, 2010; Sherman y Strang, 2004). La triangulación e integración de diferentes técnicas en ciencias sociales han demostrado ser una herramienta muy poderosa para ayudar a superar varios de los desafíos pendientes en el tema violencia penitenciaria: permiten generar mejores estimaciones descriptivas de la magnitud, variedad y gravedad del fenómeno (Eid y Eid, 2006), mejorar la conceptualización y definición (Pearce, et al., 2003) y establecer inferencias sobre sus principales causas (Seawright, 2016). No obstante, el desafío de mejorar la identificación de las causas del fenómeno también requiere acompasar la innovación metodológica con la teórica. Buena parte de las explicaciones de la violencia penitenciaria basadas en las teorías de la continuidad y la discontinuidad han estado limitadas por el uso de fuentes oficiales de datos cuantitativos. El desafío, entonces, implica utilizar la integración metodológica para continuar y profundizar la incorporación de teorías criminológicas y de otras disciplinas que han aportado a la explicación de la violencia general y adaptarlas para lograr entender la complejidad específica de la violencia penitenciaria.

Este doble desafío metodológico y teórico se vuelve particularmente relevante y útil cuando volcamos la mirada hacia la región latinoamericana. Como señalábamos al inicio, la situación de emergencia carcelaria y la ausencia de recursos, sumadas a los altos niveles de violencia y la escasa investigación, hacen urgente desarrollar la investigación en la región. De manera reciente, han empezado a desarrollarse algunos esfuerzos pioneros por emplear metodologías cuantitativas utilizando instrumentos estandarizados de autorreporte para estimar la magnitud del fenómeno de la violencia penitenciaria en varios países de la región (Bergman, 2015; Sánchez y Piñol, 2015) o evaluar las teorías de la continuidad y la discontinuidad del delito en prisión utilizando encuestas sobre calidad de vida aplicadas a personas privadas de libertad en cárceles chilenas (Sanhueza, 2015). Es imprescindible continuar desarrollando la investigación que retome las lecciones metodológicas y teóricas de literatura internacional y que las utilice para enfrentar un tercer desafío también clave en el área criminológica: el desafío cross-cultural (transcultural) (Eisner, 2014; Trajtenberg, 2017). Es un supuesto fuerte asumir que explicaciones y teorías de la violencia penitenciaria desarrolladas en Europa y Norteamérica funcionarán sin problemas cuando se las quiera aplicar a contextos tan diferentes como el latinoamericano. Por eso, el gran desafío para los próximos años es cómo desarrollar y adaptar las explicaciones de la violencia penitenciaria a las particularidades sociales, económicas, institucionales y culturales de la región, de manera tal de poder desarrollar políticas de control, mitigación y prevención de la violencia que logren ser eficaces, eficientes y justas. 


\section{Referencias bibliográficas}

Aebi, M. (2008). Temas de criminología. Estudios de criminología y política penal. Madrid: Ediciones Dykinson.

Agnew, R. (2012) Pressured into Crime: An Overview of General Strain Theory. Oxford: Oxford University Press.

Allison, M. D. y J. L. Ireland (2010). Staff and prisoner perceptions of physical and social environmental factors thought to be supportive of bullying: The role of bullying and fear of bullying. International Journal of Law and Psychiatry, 33(1), pp. 43-51.

Alzúa, M. L.; C. Rodríguez; y E. Villa (2010). The quality of life in prisons: Do educational programs reduce in-prison conflicts? En R. Di Tella, S. Edwards y E. Schargrodsky (eds.). The economics of crime: Lessons for and from Latin America, Chicago: University of Chicago Press, pp. 239-264.

Arbach-Lucioni, K.; M. Martínez-García; y A. Andrés-Pueyo (2012). Risk factors for violent behavior in prison inmates: A cross-cultural contribution. Criminal Justice and Behavior, 39(9), pp. 1219-1239.

Auty, K.; A. Cope; y A. Liebling (2017). Psychoeducational programs for reducing prison violence: A systematic review. Aggression and Violent Behavior, 33, pp. 126-143.

Auty, K. y A. Liebling (2019). Exploring the relationship between Prison Social Climate and Reoffending. Justice Quaterly. 3, pp. 1-24.

Ayete-Nyampong, L. (2015). Changing hats: Transiting between practitioner and researcher roles. En D. H. Drake, R. Earle y J. Sloan (eds.). Palgrave Handbook of Prison Ethnography. New York: Palgrave MacMillan, pp. 307-325.

Bachman, R. y J. Brent (2014). Mixed Methods. Oxford Encyclopedia of Criminology and Criminal Justice, pp. 1-6.

Bergman, M. (eds.) (2015). Estudios empiricos sobre seguridad y justicia. Informe número 2: condiciones de vida en la cárcel, resultados de la encuesta de detenidos condenados. Sáenz Peña: Universidad Nacional de Tres de Febrero.

Blevins, K. R.; S. J. Listwan; F. T. Cullen; y C. L. Jonson (2010). A general strain theory of prison violence and misconduct: An integrated model of inmate behavior. Journal of Contemporary Criminal Justice, 26(2), pp. 148-166. 
Bottoms, A. (1999). Interpersonal violence and social order in prisons. Crime and justice: A review of research, 26, pp. 205-281.

Byrne, J. y D. Hummer (2007). In search of the "Tossed Salad Man" (and others involved in prison violence): New strategies for predicting and controlling violence in prison. Aggresion and Violent Behavior, 12(5), pp. 531-541.

Comisionado Parlamentario para el Sistema Penitenciario (2009). Informe de actuación y evaluación del sistema penitenciario nacional [en línea]. Disponible en: <https://parlamento.gub.uy/sites/default/files/DocumentosCPP/ Informe2009-01.pdf $>$ [acceso 29/12/2018].

Comisionado Parlamentario para el Sistema Penitenciario (2016). Informe especial sobre muertes en prisión en 2016 [en línea]. Disponible en: $<$ https:// parlamento.gub.uy/cpp/documentos/informes-al-parlamento/86526> [acceso 29/12/2018].

Comisionado Parlamentario para el Sistema Penitenciario (2017). Informe anual 2016 [en línea]. Disponible en: <https://parlamento.gub.uy/cpp/ documentos/informes $>$ [acceso 29/12/2018].

Connell, A. y D. Farrington (1996). Bullying among incarcerated Young offenders: Developing an interview schedule and some preliminary results. Journal of Adolescence, 19(1), pp. 75-93.

Connell, A. y D. Farrington (1997). The reliability and validity of resident, staff and peer reports of bullying in young offender institutions. Psychology, Crime and Law, 3(4), pp. 287-300.

Cochran, J. C. (2012). The ties that bind or the ties that break: Examining the relationship between visitation and prisoner misconduct. Journal of Criminal Justice, 40, pp. 433-440.

Cohen, L. E. y M. Felson (1979). Social change and crime rate trends: A routine activity approach. American Sociological Review, 44, pp. 588-608.

Cooke, D. J. (1989). Containing violent prisoners: An analysis of the Barlinnie Special Unit. British Journal of Criminology, 29, pp. 129-143.

Cooke, D. J. y E. Wozniak (2010). PRISM Applied to a Critical Incident Review: A Case Study of the Glendairy Prison Riot and Its Aftermath in Barbados. International Journal of Forensic Mental Health, 9(3), pp. 159-172. 
Cooke, D. J.; E. Wozniak; y L. Johnstone (2008). Casting light on prison violence in Scotland: Evaluating the impact of situational risk factors. Criminal Justice and Behavior, 35(8), pp. 1065-1078.

Cunningham, M. D.; T. J. Reidy; y J. R. Sorensen (2005). Is death row obsolete? A decade of mainstreaming death-sentenced inmates in Missouri. Behavioral Sciences and the Law, 23(3), pp. 307-320.

Cunningham, M. D. y J. R. Sorensen (2006). Nothing to lose? A comparative examination of prison misconduct rates among life-without-parole and other long-term high-security inmates. Criminal Justice and Behavior, 33(6), pp. 683-705.

Cunningham, M. D. y J. R. Sorensen (2007). Predictive Factors for Violent Misconduct in Close Custody. The Prison Journal, 87(2), pp. 241-253.

Cunningham, M. D.; J. R. Sorensen; M. P. Vigen; y S. O. Woods (2010). Inmate homicides: Killers, victims, motives, and circumstances. Journal of Criminal Justice, 38(4), pp. 348-358.

Dachew, B.; A. Fekadu; T. Kisi; N. Yigzaw; y T. Bisetegn (2015). Psychological distress and associated factors among prisoners in North West Ethiopia: Cross-sectional study. International Journal of Mental Health Systems, 9(39), pp. 1-6.

Dammert, L. \& L. Zúñiga (2008). La cárcel: problemas y desafíos para las Américas. Santiago: FLACSO.

Davies, P. (2011). Doing interviews in prison. En P. Davies, P. Francis y V. Jupp (eds.). Doing Criminological Research. Los Ángeles: Sage Publications.

Davies, W. (2015). Unique position: Dual identities as prison researcher and exprisoner. En D. H. Drake; R. Earle y J. Sloan (eds.). Palgrave Handbook of Prison Ethnography. Londres: Palgrave MacMillan.

De León Villalba, F. (2018). Imprisonment and Human Rights in Latin America: An Introduction. The Prison Journal, 98(1), pp. 17-39.

DeLisi, M.; M. T. Berg; y A. Hochstetler (2004). Gang members, career criminals and prison violence: Further specification of the importation model of inmate behavior. Criminal Justice Studies, 17(4), pp. 369-383.

Dilulio, J. (1987). Governing Prisons: A Comparative Study of Correctional Management. NuevaYork: The Free Press. 
Dyson, G. P.; K. G. Power; y E. Wozniak (1997). Problems with using Official Records from Young Offender Institutions as Indices of Bullying. International Journal of Offender Therapy and Comparative Criminology, 41(2), pp. 121-138.

Eid, M. y D. Eid (eds.) (2006). Handbook of Multimethod Measurement in Psychology. Wahsington, DC: American Psychological Association.

Eisner, M. (2009). The Uses of Violence: An Examination of Some Cross-Cutting Issues. International Journal of Conflict and Violence, 3(1), pp. 40-59.

Eisner, M. (2014). Reducing Homicide by $50 \%$ in 30 years. Universal Mechanisms and Evidence-Based Public Policy. Artículo presentado en la Conferencia Global sobre la Reducción de la Violencia 2014. Cambridge: Organización Mundial de la Salud y Uninversidad de Cambridge.

Ellis, S. y E. Bowen (2017). Factors associated with desistance from violence in prison: an exploratory study. Psychology, Crime \& Law, 23(6), pp. 601-619.

Felson, M. y R. Boba (2010). Crime and Everyday Life. Thousand Oaks: Sage Publications.

Gadon, L.; L. Johnstone; y D. J. Cooke (2006). Situational variables and institutional violence: A systematic review of the literature. Clinical Psychology Review, 26, pp. 515-534.

Gambetta, D. (2009). Codes of the Underworld: How Criminals Communicate. Princeton: Princeton University Press.

Garcé, A.; L. Casal; C. Díaz; y L. Donnangelo (2017). Privación de libertad y reinserción social en Uruguay. Montevideo: CERES-CAF.

Gendreau, P.; C. E. Goggin; y M. A. Law (1997). Predicting prison misconducts. Criminal Justice and Behavior, 24(4), pp. 414-431.

Gesch, S. M.; S. M. Hammond; S. E. Hampson; A. Eves; y M. J. Crowder (2002). Influence of supplementary vitamins, minerals and essential fatty acids on the antisocial behavior of young adult prisoners: Randomised, placebocontrolled trial. British Journal of Psychiatry, 181(1), pp. 22-28.

Giordano, P. C.; S. A. Cernkovich; y J. L. Rudolph (2002). Gender, crime and desistance: Toward a theory of cognitive transformation. American Journal of Sociology, 107(4), pp. 990-1064.

Gottfredson, M. y T. Hirschi (1990). A General Theory of Crime. Stanford, CA: Stanford University Press. 
Grasmick, H. G.; C. R. Tittle; R. J. Bursik; y B. J. Arneklev (1993). Testing the Core Empirical Implications of Gottfredson and Hirschi's General Theory of Crime. Journal of Research in Crime and Delinquency, 30(1), pp. 5-29.

Harvey, J. (2015). The Ethnographic Practitioner. En D. H. Drake, R. Earle y J. Sloan (eds.). Palgrave Handbook of Prison Ethnography. Nueva Jersey: Palgrave MacMillan.

Hindelang, M. J.; M. R. Gottfredson; y J. Garofalo (1978). Victims of personal crime: An empirical foundation for a theory of personal victimization. Cambridge, MA: Ballinger.

Hirschi, T. (1969). Causes of Delinquency. Berkeley, CA: University of California Press.

Homel, R. y C. Thomson (2005). Causes and prevention of violence in prisons. En S. O’Toole y S. Eyland (eds.). Sidney: Hawkins Press. Corrections criminology, pp. 101-108.

Imbush, P.; M. Misse y F. Carri (2011). Violence research in Latin America and the Caribbean: A literature review. International Journal of Conflict and Violence, 5(1), pp. 87-154.

International Centre for Prison Studies (2018). World Prison Brief [en línea]. Disponible en: <http://www.prisonstudies.org > [acceso: 09/03/2018]

Ireland J. L. (1998). Direct and Indirect Prisoner Behaviour Checklist (DIPC). Preston: University of Central Lancashire.

Ireland, J. L. (1999). Bullying behaviors among male and female prisoners: A study of adult and young offenders. Aggressive Behavior, 25(3), pp. 161178.

Ireland, J. L. (2002). Bullying among prisoners. Evidence, Research and Intervention Strategies. Londres y Nueva York: Routledge Taylor and Francis Group.

Ireland, J. L. (2012). Understanding bullying among younger prisoners: recent research and introducing the multifactor model of bullying in secure settings. International Journal of Adolescent Medicine and Health, 24(1), pp. 63-8.

Ireland, J. L. y J. Archer (1996). Descriptive analysis of bullying in male and female adult prisoners. Journal of Community and Applied Social Psychology, 6, pp. 35-47. 
Ireland, C. A. y J. L. Ireland (2000). Descriptive analysis of the nature and extent of bullying behavior in a maximum-security prison. Aggressive Behavior, 26(3), pp. 213-223.

Irwin, J. K. y D. R. Cressey (1962). Thieves, convicts and the inmate culture. Social Problems, 10, pp. 142-155.

Jackson, J.; C. Heard; y H. Fair (2017). Prison. Evidence of its use and over use from around the world. Londres: Institute for Criminal Policy Research, Birkbeck, University of London.

Jackson, J.; T. Tyler; B. Bradford; D. Taylor; y M. Shiner (2010). Legitimacy and procedural justice in prisons. Prison Service Journal, 191, pp. 4-10.

Johnstone, L. y D. J. Cooke (2008). PRISM: Promoting Risk Intervention by Situational Management. Structured professional guidelines for assessing situational risk factors for violence in institutions. Burnaby: Simon Fraser University, Mental Health, Law, and Policy Institute.

Juanche, A. (2012). La población adulta privada de libertad. En A. Juanche y J. Palummo (coords.). Hacia una política de Estado en privación de libertad. Diálogo, recomendaciones y propuestas. Montevideo: Observatorio del Sistema Judicial, Servicio de Paz y Justicia, pp. 89-202.

Junger-Tas, J. y I. Haen Marshall (1999). The Self-Report Methodology in Crime Research. Crime and Justice, 25, pp. 291-367.

Kerley, K.; A. Hochstetler; y H. Copes (2009). Self-Control, Prison Victimization and Prison Infractions. Criminal Justice Review, 34(4), pp. 553-568.

Kerley, K.; H. Copes; R. Tewksbury; y D. Dabney (2011). Examining the Relationship between Religiosity and Self-Control as Predictors of Prison Deviance. International Journal of Offender Therapy and Comparative Criminology, 55(8), pp. 1251-1271.

Kidd, R. F. y E. F. Chayet (2010). Why Do Victims Fail to Report? The Psychology of Criminal Victimization. Journal of Social Issues, 40(1), pp. 39-50.

Krebs, J. R. y N. B. Davis (1993). An Introduction to Behavioral Ecology. Oxford: Blackwell.

Krug, E. G.; L. L. Dahlberg; J. A. Mercy; A. B. Zwi; y R. Lozano (2003). Informe mundial sobre la violencia y la salud. Washington DC: Organización Panamericana de la Salud, Oficina Regional para las Américas. 
Lahm, K. (2009). Inmate Assaults on Prison Staff. A Multilevel Examination of an Overlooked Form of Prison Violence. The Prison Journal, 89(2), pp. 131-150.

Laub, J. H. y R. J. Sampson (2001). Understanding desistance from crime. Crime and justice, 28, pp. 1-69.

Laub, J. H.; D. S. Nagin; y R. J. Sampson (1998). Trajectories of change in criminal offending: Good marriages and the desistance process. American Sociological Review, 63(2), pp. 225-238.

Lessing, B. (2017). Counterproductive punishment: How prison gangs undermine state authority. Rationality and Society, 29(3), pp. 257-297.

Liebling, A.; S. Hulley; y B. Crewe (2012). Conceptualising and measuring the quality of prison life. En D. Gadd, S. Karstedt y S. F. Messner (eds.). The Sage handbook of criminological research methods. Londres: Sage, pp. 358-372.

Maguire, M. (2012). Criminal Statistics and the Construction of Crime. En M. Maguire y R. Morgan (eds.). The Oxford Handbook of Criminology. Oxford: Oxford University Press, pp. 206-244

Marquart, J.W. (1986). Doing research in prison: the strengths and weaknesses of full participation as a guard. Justice Quarterly, 3(1), pp. 15-32.

Maruna, S. (2001). Making good: How ex-convicts reform and rebuild their lives. Washington DC: American Psychological Association Books.

Maruna, S. (2010). Mixed Method Research in Criminology: Why Not Go Both Ways? En A. Piquero y D. Weisburd (eds.). Handbook of Quantitative Criminology. Nueva York: Springer, pp. 123-140.

Matthews, R. (2012). Doing time: An introduction to sociology of Punishment. Londres: Palgrave MacMillan.

Maxfield, M. G. y E. R. Babbie (2015). Research Methods for Criminal Justice and Criminology. Stamford: Cengage Learning.

McCorkle, R.; T. D. Miethe; y K. A. Drass (1995). The roots of prison violence: A test of the deprivation, management, and "not-so-total" institution models. Crime \& Delinquency, 41(3), pp. 317-331.

McGuire, J. (2008). A Review of Effective Interventions for Reducing Aggression and Violence. Philosophical Transactions of the Royal Society B: Biological Sciences, 363(1503), pp. 2577-2597. 
McGuire, J. (2018). Understanding Prison Violence: A Rapid Assessment. Analytical Report, HM Prison \& Probation Service [en línea]. Disponible en: <https://assets.publishing.service.gov.uk/government/uploads/system/ uploads/attachment_data/file/737956/understanding-prison-violence.pdf> [acceso 15/12/2018].

Mears, D. P.; E. A. Stewart; S. E. Siennick; y R. L. Simons (2013). The code of the street and inmate violence: Investigating the salience of imported belief systems. Criminology, 51(3), pp. 695-728.

Modvig, J. (2014). Violence, sexual abuse and torture in prisons. En S. Enggist, L. Møller y C. Udesen (eds.). Prisons and Health. Copenhague: Organización Mundial de la Salud Región Europa, pp. 19-26.

Mooney, J. L. y M. Daffern (2015). The relationship between aggressive behaviour in prison and violent offending following release. Psychology, Crime \& Law, 21(4), pp. 314-329.

Moos, R. H. (1975). Evaluating correctional and community settings. Nueva York: Wiley.

Nowak, M. (2010). Informe del Relator Especial sobre la tortura y otros tratos o penas crueles, inhumanos o degradantes. Misión a Uruguay. Montevideo: Naciones Unidas Uruguay.

Oficina de Naciones Unidas contra la Droga y el Delito (2014). The Global Study on Homicide 2013: Trends, Contexts, Data. Vienna: United Nations Office for the Coordination of Humanitarians Affairs.

Organización Mundial de la Salud (2002). World Report on Violence and Health: Summary. Ginebra: Organización Mundial de la Salud.

Pearce, C. L.; H. P. Sims; J. F. Cox; G. Ball; E. Schnell; K. A. Smith; y L. Trevino (2003). Transactors, transformers and Beyond: A multi-method develompent of a theoretical typology of leadership. The Journal of Managment Development, 22(4), pp. 273-307.

Pierce, M. B.; T. L. Freiburger; J. R. Chapin; E. Brittany; y T. Madden (2018). Assessing the impact of visitation on inmate misconduct within a county jail. Security Journal, 31(1), pp. 1-20.

Porporino, F. J.; P. D. Doherty; y T. Sawatsky (1987). Characteristics of homicide victims and victimization in prisons: A Canadian historical perspective. International Journal of Offender Therapy and Comparative Criminology, 31(2), pp. 125-136. 
Redondo, S. (2008). Individuos, sociedades y oportunidades en la explicación y prevención del delito: Modelo del Triple Riesgo Delictivo (TRD). Revista Española de Investigación Criminológica, (7)6, pp. 1-59.

Reisig, M. D. (1998). Rates of disorder in higher-custody state prisons: A comparative analysis of managerial practices. Crime and Delinquency, 44, pp. 229-244.

Rocheleau, A. M. (2013). An Empirical Exploration of the "Pains of Imprisonment" and the Level of Prison Misconduct and Violence. Criminal Justice Review, 38(3), pp. 354-374.

Rosenberg, A. (2012). Philosophy of Social Science. Filadelfia: Westview Press.

Sampson, R. y J. Laub (1993). Crime in the Making: Pathways and Turning Points Through Life. Cambridge: Harvard University Press.

Sánchez, M. y D. Piñol (2015). Condiciones de vida en los centros de privación de libertad en Chile. Análisis a partir de una encuesta aplicada a siete países de Latinoamérica. Santiago: Centros de Estudios Públicos en Seguridad Ciudadana.

Sanhueza, G.; M. A. Smith; y V. Valenzuela (2015). Victimización física entre internos en cárceles chilenas: una primera exploración. Revista de Trabajo Social, 88, pp. 61-73.

Schenk, A. y W. Fremouw (2012). Individual characteristics related to prison violence: A critical review of the literature. Aggression and Violent Behavior, 17, pp. 430-442.

Seawright, J. (2016). Multi-Method Social Science. Combining Qualitative and Quantitative Tools. Cambridge: Cambridge University Press.

Servicio de Paz y Justicia (2012). Hacia una política de Estado en privación de libertad. Montevideo: Servicio de Paz y Justicia.

Sheeran, P. (2012). Intention-Behavior Relations: A Conceptual and Empirical Review. European Review of Social Psyhcology, 12(1), pp. 1-21.

Sherman, L. y H. Strang (2004). Experimental Ethnography: The Marriage of Qualitative and Quantitative Research. The Annals of the American Academy of Political and Social Science, 595(1), pp. 204-222.

Sorensen, J. R. y M. D. Cunningham (2007). Operationalizing risk: The influence of measurement choice on the prevalence and correlates of prison violence among incarcerated murderers. Journal of Criminal Justice, 35(5), pp. 546-555. 
Sorensen, J. y M. D. Cunningham (2010). Conviction Offense and Prison Violence A Comparative Study of Murderers and Other Offenders. Crime \& Delinquency, 56(1), pp. 103-125.

Sorensen, J.; M. D. Cunningham; M. P. Vigen; y S. O. Wood (2011). Serious assaults on prison staff: A descriptive analysis. Journal of Criminal Justice, 39, pp. 143-150.

Steiner, B. (2009). Assessing static and dynamic influences on inmate violence levels. Crime \& Delinquency, 55(1), pp. 134-161.

Steiner, B.; D. Butler; y J. M. Ellison (2014). Causes and correlates of prison inmate misconduct: A systematic review of the evidence. Journal of Criminal Justice, 42(6), pp. 462-470.

Steiner, B. y C. M. Cain (2016). The Relationship between Inmate Misconduct, Institutional Violence, and Administrative Segregation: A Systematic Review of the Evidence. NCJ 250320. En National Institute of Justice (2016), Restrictive Housing in the United State: Issues, Challenges, and Future Directions. Washington DC: US Department of Justice, National Institute of Justice.

Steiner, B.; J. M. Ellison; H. D. Butler; y C. M. Cain (2017). The Impact of Inmate and Prison Characteristics on Prisoner Victimization. Trauma, Violence, \& Abuse, 18(1), pp. 17-36.

Steiner, B. y J. Wooldredge (2009). Individual and environmental effects on assaults and nonviolent rule-breaking by women in prison. Journal of Research in Crime and Delinquency, 46(4), pp. 437-467.

Sutton J. E. (2011). An ethnographic account of doing survey research in prison: descriptions, reflections, and suggestions from the field. Qualitative Sociology Review, VII(2), pp. 45-63.

Sykes, G. M. (1958). The society of captives: A study of a maximum security prison. Princeton, NJ: Princeton University Press.

Toman, E. L.; J. C. Cochran; J. K. Cochran; y W. Bales (2015). The implications of sentence length for inmate adjustment to prison life. Journal of Criminal Justice, 43(6), pp- 510-521.

Trajtenberg, N. (2017). A matter of costs and benefits? The role of morality, legitimacy and self-control as moderators of the link between rationality and youth delinquency in Uruguay. Disertación de doctorado en Criminología. Cambridge: University of Cambridge. 
Trulson, C. R.; M. DeLisi; y J. W. Marquart (2011). Institutional misconduct, delinquent background, and rearrest frequency among serious and violent delinquent offenders. Crime \& Delinquency, 57(5), pp. 709-731.

Ugelvik, T. (2014). Prison Ethnography as Lived Experience: Notes From the Diaries of a Beginner Let Loose in Oslo Prison. Qualitative Inquiry, 20(4), pp. 471-480.

Useem, B. y A. M. Piehl (2006). Prison buildup and disorder. Punishment \& Society, 8(1), pp. 87-115.

Useem, B. y M. Reisig (1999). Collective action in prisons: Protests, disturbances, and riots. Criminology, 37(4), pp. 735-759.

Vigna, A. y S. Sosa Barón (2019). Muertes en las cárceles uruguayas. Magnitud del fenómeno y problemas para estudiarlo. Revista de Ciencias Sociales, 32(45), pp. 39-66.

Walters, G. D. (1998). Time series and correlational analysis of inmate-initiated assaultive incidents in a large correctional system. International Journal of Offender Therapy and Comparative. Criminology, 42, pp. 124-132.

Walters, G. D. (2003). Changes in criminal thinking and identity in novice and experienced inmates: Prisonization revisited. Criminal Justice and Behavior, 30(4), pp. 399-421.

Walters, G. D. (2011). Criminal thinking as a mediator of the mental illnessprison violence relationship: A path analytic study and causal mediation analysis. Psychological Services, 8(3), pp. 189-199.

Waters, J. E. y W. L. Megathlin (2002). Evaluating Change in Social Climate in a Close Security State Correctional Facility. Journal of Offender Rehabilitation, 34(4), pp. 71-84.

Wikström, P. O. H.; D. Oberwittler; K. Treiber; y B. Hardie (2012). Breaking Rules: The Social and Situational Dynamics of Young People's Urban Crime. Oxford: Oxford University Press.

Willis, G. D. (2014). The Killing Consensus: Police, Organized Crime, and the Regulation of Life and Death in Urban Brazil. Berkeley: University of California Press.

Wolff, N.; J. Shi y R. Bachman (2008). Measuring Victimization inside Prisons: Questioning the Questions. Journal of Interpersonal Violence, 23(10), pp. 1343-1362. 
Wolff, N.; C. L. Blitz; J. Shi; J. Siegel; y R. Bachman (2007). Physical violence inside prisons: Rates of victimization. Criminal Justice and Behavior, 34(5), pp. 588-599.

Wooldredge, J. y B. Steiner (2013). Violent Victimization among State Prison Inmates. Violence and Victims, 28(3), pp. 531-551.

Wooldredge, J.; T. Griffin y T. Pratt (2001). Considering Hierarchical Models for Research on Inmate Behavior: Predicting Misconduct with Multilevel Data. Justice Quarterly, 18, pp. 203-231.

\section{Contribución de autoría}

Nicolás Trajtenberg contribuyó en un $60 \%$ a la redacción de este artículo, mientras que Olga Sánchez de Ribera lo hizo en un $40 \%$. 